\title{
BLOCK-BASED LOSSLESS DATA HIDING IN DELTA DOMAIN
}

\author{
Shu-Kei Yip, Oscar C. Au, Hoi-Ming Wong, Chi-Wang Ho \\ Department of Electrical and Electronic Engineering \\ The Hong Kong University of Science and Technology \\ Clear Water Bay, Hong Kong \\ Email: \{sukiyip, eeau, jodyho, hoimingw\}@ust.hk
}

\begin{abstract}
Digital watermarking is one of the ways to prove the ownership and the authenticity of the media. However, some applications, such as medical and military, are sensitive to distortion, this highlights the needs of lossless watermarking. In this paper, we propose a new lossless data hiding algorithm in delta domain. A MSE discount is obtained by using checkerboard-pattern watermark sequences. The PSNR between the watermarked image and the original image is high and there is no "salt-and-peppers" artifact. The proposed algorithm can be extended to withstand the JPEG attack.
\end{abstract}

\section{INTRODUCTION}

Digital watermarking is one of the solutions to protect intellectual properties and copyright by hiding information into digital media. In order to enhance the security of watermark, the watermark should be perceptually transparent and robustness [1] [2]. Distortion is usually introduced into host image during watermark embedding process and results in Peak Signal-to-Noise Ratio (PSNR) loss. Although the distortion is normally small, some applications, such as medical and military, are sensitive to the embedding distortion and prohibit permanent loss of signal fidelity. This highlights the necessary for lossless/ reversible watermarking. Lossless watermarking is a technique to embed watermark data into a host image, and the original host signal can be recovered perfectly after the watermark extraction process.

There are many existing lossless watermarking algorithms. To the best of our knowledge, the concept of lossless invisible watermarks first appeared in a patent owned by C.W. Honsinger et al. [3]. Modulo operations are used to ensure the reversibility, however, it may result in "salt-and-peppers" artifacts. C. De Vleeschouwer et al. proposed a circular interpretation of bijective transform for lossless watermarking [4]. Although the algorithm can withstand some degree of the JPEG attack, "salt-and-peppers" artifacts is the major disadvantage of the algorithm. Adnan M. Alattar proposed using the prediction error between the predicted pixel value and the original pixel value to embed data, however, some overhead is needed to ensure the reversibility [5].

In this paper, we propose a new lossless data hiding algorithm in delta domain. Watermark is embedded in the general smooth region (low variance region), and the strong edge is preserved. The Peak Signal-to-Noise Ratio (PSNR) between the watermarked image and original host image is high and there is no "salt-andpeppers" artifact as no modulo operation is involved. A MeanSquare Error (MSE) discount is obtained by embedding the watermark in the proposed delta domain when compare with [4]. Using the checkerboard-pattern watermark sequences and the delta domain, half of the total pixels in the image remain unchanged after watermark embedding. The proposed algorithm can be extended to withstand the JPEG attack by storing the location map to the authorized party.

This paper is organized as follows. In section 2, the details of the proposed data hiding algorithm is presented. Apart from that, we will also investigate the performance of our proposed algorithm. Section 3 shows the experimental results, such as PSNR between the watermarked image and the original image, and the payload. In section 4 , we present the general idea about the extension of our algorithm to withstand the JPEG attack and show the Bit Error Rate (BER) of the extracted logo when compared with the original logo. And there is a conclusion in section 5 .

\section{PROPOSED DATA HIDING ALGORITHM}

In this section, we introduce the details of the delta domain in section 2.1. The principle and the details of watermark embedding and watermark extraction are presented in section 2.2 and 2.3 respectively. In section 2.4, the performance analysis of our proposed algorithm, such as the lower bound of PSNR and the reversibility of our proposed data hiding algorithm, are investigated.

Let us denote the original host image as $P$ with the size of $P_{X} \times P_{Y}, P(x, y)$ be the pixel value of $P$ at $(x, y)$, where $x=1 \ldots P_{X}, y$ $=1 \ldots P_{Y}, P(x, y) \in\{1 \ldots 255\}$. A binary watermark, $W$, is embedded into $P$ to form a watermarked image, $Q$.

\subsection{Delta Domain}

In this paper, we propose a new domain called delta domain. Delta domain can be interpreted as the vertical high-pass of $P$. The delta domain of $P, D$, with the same size as $P$, can be formed by using the following delta transform equation:

$$
D(x, y)=\left\{\begin{array}{lr}
P(x, y) & \text { if } y=1 \\
P(x, y)-P(x, y-1) & \text { if } y \geq 2
\end{array}\right.
$$

The first row of $D, D(x, 1)$, is used to reconstruct the image from delta domain and to be a reference when performing inverse delta transform, and $D(x, 1)$ is not used for data hiding. For $y \geq 2$, the larger the value of $D$ is, the sharper the gradient change in pixel value in spatial domain is. By examining the value of $D$, we can embed the watermark in a generally smooth region and preserve the edge property of the image.

\subsection{Watermark Embedding Process}

After the formation of delta domain, $D$ is divided into $B_{X} \times B_{Y}$ blocks (the first row is not used). Let us denotes the block set which is suitable for data hiding as SSet and the block set which is 
not suitable for data hiding as NSet. For each block, $B$, it is further subdivided into sub-blocks, $S$, with the size of $S_{X} \times S_{Y} S_{X}$ and $S_{Y}$ should be an even number in order to achieve the MSE discount.

To perform watermarking, a set of condition matrix, $V_{C}$, is used to check whether the blocks are qualified to be in SSet. By using a pair of watermark sequences with checkerboard-pattern and spread spectrum technique, we can embed the watermark into the digital image with MSE discount. The details of formation of condition matrix and watermark sequence pair are shown in section 2.2.1. In section 2.2.2, we present the block condition checking. Block condition checking is used to prevent "salt-andpeppers" artifacts and to ensure there are more than $N_{\min }$ subblocks in a block which are "unbiased" to the watermark sequences pair before watermark embedding. Section 2.2.3 describes the details of watermark embedding. Figure 1 shows the flow chart of watermark embedding.

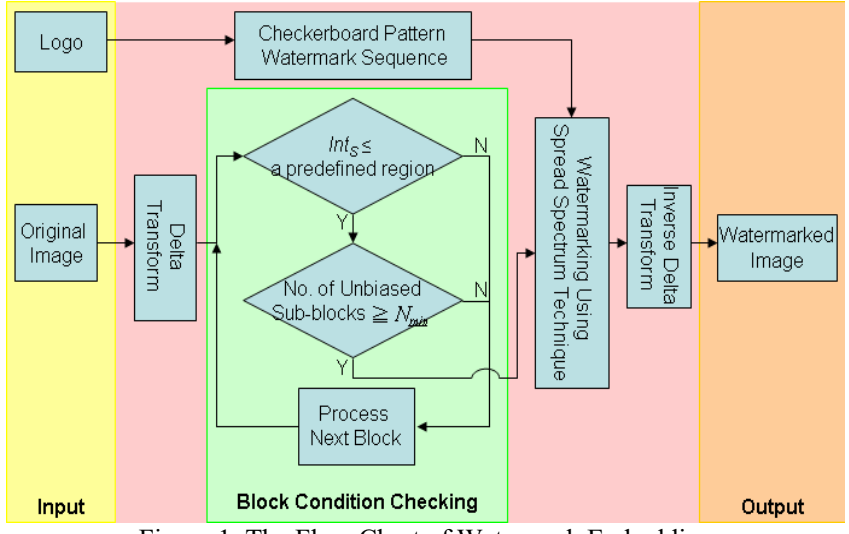

Figure 1. The Flow Chart of Watermark Embedding

\subsubsection{Formation of Condition Matrix and Watermark Sequences Pair}

Let us denote the inner product of $A$ and $B$ as $\langle A, B\rangle$ :

$$
<A, B>\triangleq \frac{1}{N N} \sum_{i=1}^{N} \sum_{j=1}^{N} A(i, j) B(i, j)
$$

For binary watermark, if watermark bit is " 0 " (" 1 "), a watermark sequence, $W_{\text {seql }}\left(W_{\text {seq } 2}\right)$, is used. There are three requirements for the watermark sequences pair (W1-3).

W1) $W_{\text {seq } 1}$ and $W_{\text {seq2 } 2}$ are complement pair.

W2) $W_{\text {seq } 1}$ and $W_{\text {seq } 2}$ are the same size as $S$.

W3) The "1"s and the "- 1 "s in the $W_{\text {seql }}$ and $W_{\text {seq2 }}$ are the same and with checkerboard-pattern.

In our proposed algorithm, there are $N_{C}$ numbers of condition matrix, $V_{C}$, and there are mainly three requirements for $V_{C}(\mathrm{R} 1-3)$ :

R1) There are the same number of "1"s and "-1"s in each $V_{C}$.

R2) $<W_{\text {seq }}, V_{C}>=0$.

R3) Each $V_{C}$ is orthogonal to each others, $\left\langle V_{C i}, V_{C j}\right\rangle=0$ for $i \neq j$.

Using $2 \times 2$ sub-block as an example, and $N_{C}$ is set to 2 , one of the possible set of $V_{C}, V_{C l}$ and $V_{C 2}$ are:

$$
V_{C 1}=\left[\begin{array}{cc}
1 & 1 \\
-1 & -1
\end{array}\right] \quad V_{C 2}=\left[\begin{array}{ll}
1 & -1 \\
1 & -1
\end{array}\right]
$$

,and the pair of watermark sequences, $W_{\text {seq } 1}$ and $W_{\text {seq2 } 2}$, are:

$$
W_{\text {seq } 1}=\left[\begin{array}{cc}
1 & -1 \\
-1 & 1
\end{array}\right] \quad W_{\text {seq } 2}=\left[\begin{array}{cc}
-1 & 1 \\
1 & -1
\end{array}\right]
$$

\subsubsection{Block Condition Checking}

After the formation of $V_{C}$ and $W_{\text {seq }}$, block condition checking is followed. There are two conditions checking (C1-2) to check whether the block belongs to SSet:

C1) For all the sub-blocks, the corresponding average spatial intensity value, Int $_{S}$, should be within a predefined range.

C2) There should be more than $N_{\min }$ sub-blocks which $<S, V_{C}>\leq T$, where $T$ is a predefined $2 \mathrm{D}$ region.

The constraint of $(\mathrm{C} 1)$ is used to prevent the "salt-andpeppers" artifacts. For the constraint of $(\mathrm{C} 2)$, it is to look for the blocks which have more sub-blocks less correlated with $W_{\text {seq }}$, and to ensure there are more than $N_{\min }$ sub-blocks in a block are the "unbiased" sub-blocks to the $W_{\text {seq }}$. Moreover, $(\mathrm{C} 2)$ is to ensure the sub-blocks are generally smooth region. For the blocks which do not belong to SSet, they are in NSet.

\subsubsection{Watermark Embedding}

According to the watermark bit, $W_{\text {seq } 1}$ or $W_{\text {seq } 2}$ is watermarked to all sub-blocks in the block which belongs to SSet using the following equation.

$$
S^{\prime}=S+\Delta W_{\text {seq }} \text { where seq }=\{\text { seq } 1, \text { seq } 2\}
$$

For the sub-blocks which belong to NSet:

$$
S^{\prime}=S
$$

$S^{\prime}$ is the watermarked sub-block coefficients in delta domain and $\Delta$ is the watermark strength and should be an integer for ensuring reversibility. If the block is in NSet, which means there is a strong edge across many sub-blocks or the block is with high texture property so that the block is not suitable to embed watermark in order to preserve the edge property. The watermarked image, $Q$, is formed by performing watermark embedding using (3) or (4) in delta domain followed by the inverse delta transform. The inverse delta transform is shown as follows:

$$
Q(x, y)= \begin{cases}D(x, y) & \text { if } y=1 \\ Q(x, y-1)+D^{\prime}(x, y) & \text { if } y \geq 2\end{cases}
$$

\subsection{Watermark Extraction Process}

Watermark extraction is performed by checking the received image with condition (C1) and (C2) using the same set of $V_{C}$ and $W_{\text {seq1 }}$ and $W_{\text {seq2 }}$. Because of the number of "1"s and "-1"s in the watermark sequences are the same, Int $t_{S}$ is preserved after watermark embedding if there is no attack and the mathematical proof is shown as follows.

$$
\begin{aligned}
\text { Int }_{S} & =\frac{1}{S_{X} S_{y}} \sum_{i=1}^{S_{X}} \sum_{j=1}^{S_{Y}} Q(\alpha+i, \beta+j) \\
& =\frac{1}{S_{X} S_{y}} \sum_{i=1}^{S_{X}} \sum_{j=1}^{S_{Y}}\left(Q(\alpha+i, \beta+j-1)+D^{\prime}(\alpha+i, \beta+j)\right) \\
& \left.=\frac{1}{S_{X} S_{y}} \sum_{i=1}^{S_{X}} \sum_{j=1}^{S_{Y}}\left(Q(\alpha+i, \beta+j-1)+D(\alpha+i, \beta+j)+\Delta W_{\text {seq }}(i, j)\right)\right) \\
& =\frac{1}{S_{X} S_{y}} \sum_{i=1}^{S_{X}} \sum_{j=1}^{S_{Y}}(Q(\alpha+i, \beta+j-1)+D(\alpha+i, \beta+j)) \because \sum_{x=1}^{S_{X}} \sum_{y=1}^{S_{Y}} W_{\text {seq }}(i, j)=0 \\
& =\frac{1}{S_{X} S_{y}} \sum_{i=1}^{S_{X}} \sum_{j=1}^{S_{Y}} P(\alpha+i, \beta+j)
\end{aligned}
$$$$
\alpha \text { and } \beta \text { are the starting index of sub-block, } S
$$ 
For (C2), because of the (R2), $\left\langle S^{\prime}, V_{C}>=<S, V_{C^{\prime}}\right.$ :

$$
\begin{aligned}
<S^{\prime}, V_{C}> & =<S+\Delta W, V_{C}> \\
& =<S, V_{C}>+<\Delta W, V_{C}>
\end{aligned}
$$$$
=\left\langle S, V_{C}\right\rangle
$$

For NSet, as there is no change, it is obvious that the results of (C1-2) will be the same as watermark embedding. The watermark bit decision for a sub-block is shown in figure 2. For the blocks belonging to SSet, each block $S$ is inner product or correlation with $W_{\text {seq } 1}$ and $W_{\text {seq2 } 2}$. The result of $\left\langle S^{\prime}, W_{\text {seq }}\right\rangle$ with 2 watermark sequences are computed. The bit decision is shown as follows:

$$
W= \begin{cases}0 & \text { if }<\mathrm{W}_{\text {seq } 1}, S^{\prime}>><\mathrm{W}_{\text {seq2 }}, S^{\prime}> \\ 1 & \text { if }<\mathrm{W}_{\text {seq1 } 1}, S^{\prime}>\leq<\mathrm{W}_{\text {seq2 } 2}, S^{\prime}>\end{cases}
$$

The extracted watermark bit of a block, $B$, is based on the voting of all the sub-blocks' decoding results. As there are more than $N_{\min }$ sub-blocks are "unbiased" to the watermark sequences in each block, the watermark can be decoded accurately using voting.

We can interpret the inner product as projection. By projecting the sub-block, $S$, in delta domain on the $V_{C}$, we can decide the watermark embedded in each sub-block. The projection is shown in figure 2.

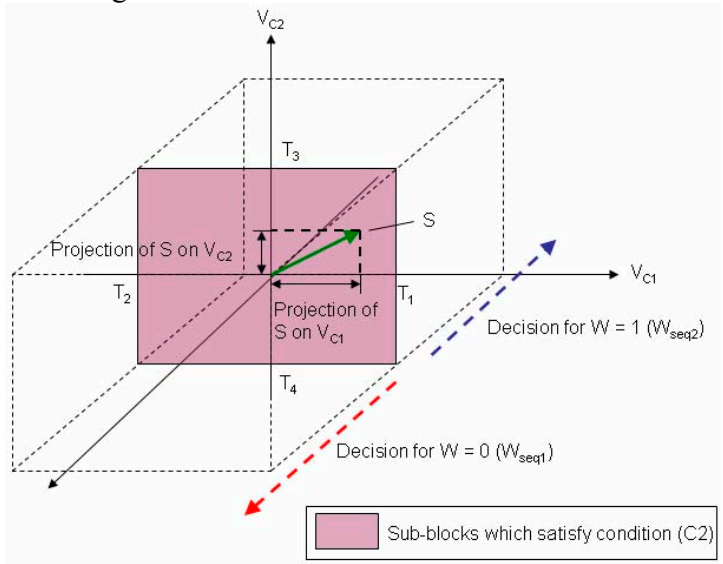

Figure 2. Projections of $S$ onto $V_{C l}$ and $V_{C 2}\left(N_{C}=2\right)$

\subsection{Performance Analysis of Our Proposed Algorithm}

The upper bound of the payload of our proposed data hiding algorithm depends on the block size, the image size and the image property. The upper bound of payload is the total number of blocks, $B$, in the image.

The lower bound of PSNR depends on the watermark strength, $\Delta$. As we modify the elements in delta domain, half of the pixels of each sub-block will not be changed. The mathematical proof is shown as follows:

$$
\begin{aligned}
Q & (x, y)=Q(x, y-1)+D^{\prime}(x, y) \\
& =Q(\alpha+i, \beta+j-1)+D(\alpha+i, \beta+j)+\Delta W_{\text {seq }}(i, j) \\
& =\left\{\begin{array}{cc}
Q(\alpha+i, \beta+j-1)+D(\alpha+i, \beta+j)+\Delta W_{\text {seq }}(i, j) & \text { if } \mathrm{i} \text { is odd } \\
Q(\alpha+i, \beta+j-2)+D(\alpha+i, \beta+j-1)+D(\alpha+i, \beta+j) & \text { if } \mathrm{j} \text { is even } \\
+\Delta W_{\text {seq }}(i, j-1)+\Delta W_{\text {seq }}(i, j)
\end{array}\right. \\
\because & W_{\text {seq }} \text { is in checkerboard pattern } \\
\quad & \left\{\begin{array}{cc}
Q(\alpha+i, \beta+j-1)+D(\alpha+i, \beta+j)+\Delta W_{\text {seq }}(i, j) & \text { if } \mathrm{i} \text { is odd } \\
Q(\alpha+i, \beta+j-2)+D(\alpha+i, \beta+j-1)+D(\alpha+i, \beta+j) & \text { if } \mathrm{j} \text { is even }
\end{array}\right. \\
\quad & \left\{\begin{array}{cc}
P(\alpha+i, \beta+j)+\Delta W_{\text {seq }}(i, j) & \text { if } \mathrm{i} \text { is odd } \\
P(\alpha+i, \beta+j) & \text { if } \mathrm{j} \text { is even }
\end{array}\right.
\end{aligned}
$$

From the above equation, when the sub-block index, $j$, is even (which is the even-row of each sub-block), the watermarked pixel value is the same as the original pixel value. As a result, there are half of the pixels in the SSet are remained unchanged, and the MSE discount of SSet is:

$$
\text { MSEdiscount }=\frac{1}{2} \Delta \times \Delta
$$

For the blocks of NSet, all the pixels remain unchanged. As a result, the general expression of lower bound of PSNR of our data hiding algorithm is:

$$
P S N R \geq 10 \log \frac{255 \times 255}{0.5 \Delta \times \Delta}
$$

\section{EXPERIMENTAL RESULTS}

We have tested the proposed data hiding algorithm with several standard bitmap testing images. They are Lena, Barbara, Baboon, F16, Fishingboat and Peppers. All the image are $512 \times 512$. We divide the images into $8 \times 8$ block and $2 \times 2$ sub-block. The $N_{\min }$ is set to 7 and $\Delta$ is set as 16 . The original Lena, the watermarked Lena with 3509 bits and the embedded logo are shown in figure 3.
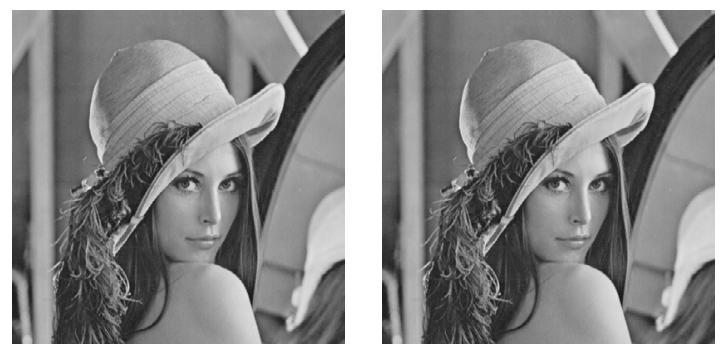

HK UST

Figure 3. Original Lena (left), Watermarked Lena (3213 bits) (Middle) and the HKSUT Logo (Right)

The comparisons of the PSNR (in $\mathrm{dB}$ ) between the watermarked images and the original images and payload (in bitper-pixel) of our proposed algorithm with that of [4] are shown in table 1.

Table 1. PSNR and Payload of Different Images

\begin{tabular}{|l|c|c|c|c|}
\hline Image & $\begin{array}{l}\text { Payload of } \\
\text { our } \\
\text { proposed } \\
\text { algorithm }\end{array}$ & $\begin{array}{l}\text { PSNR of } \\
\text { our } \\
\text { proposed } \\
\text { algorithm }\end{array}$ & $\begin{array}{l}\text { Payload } \\
\text { of [4] }\end{array}$ & $\begin{array}{l}\text { PSNR } \\
\text { of [4] }\end{array}$ \\
\hline Lena & 0.0134 & 28.55 & 0.0135 & 24.04 \\
\hline Barbara & 0.0103 & 29.48 & 0.0117 & 24.04 \\
\hline Baboon & 0.0032 & 36.16 & 0.0091 & 23.29 \\
\hline F16 & 0.0128 & 28.68 & 0.0139 & 24.05 \\
\hline Fishingboat & 0.0111 & 30.05 & 0.0127 & 20.21 \\
\hline Peppers & 0.0105 & 30.45 & 0.0119 & 23.75 \\
\hline
\end{tabular}

From table 1, the payload is around $0.01 \mathrm{bpp}$ in most cases, which is similar to [4], however, the PSNR of our proposed algorithm is much higher than that of [4]. Using equation (8), the lower bound of our proposed algorithm using the testing condition is $27.06 \mathrm{~dB}$, and this bound is verified by table 1 . For [4], the upper bound of PSNR (as modulo operation is not taken into account which will result in "salt-and-peppers" artifacts) of [4] is shown in the following equation.

$$
P S N R \leq 10 \log \frac{255 \times 255}{\Delta \times \Delta}
$$


By using the same testing condition, the upper bound of PSNR of [4] is $24.05 \mathrm{~dB}$, which is lower than the lower bound of our proposed algorithm.

The relationship between the payload (bpp) and the PSNR (dB) of Lena using the proposed algorithm is shown in figure 4. The larger the $N_{\min }$ is, the smaller the payload is, and the higher the PSNR is.

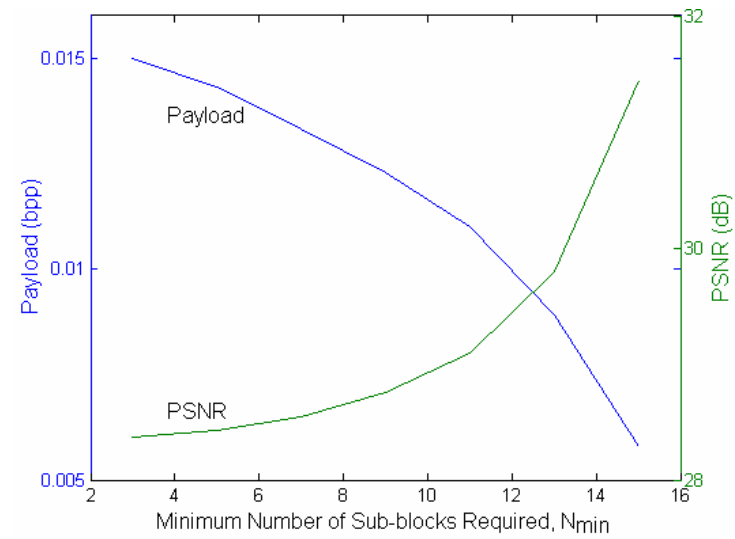

Figure 4. Payload and PSNR with Different $N_{\text {min }}$

The embedding location of Lena is shown in figure 5. The white blocks mean the blocks belong to NSet, and the other blocks belong to SSet.

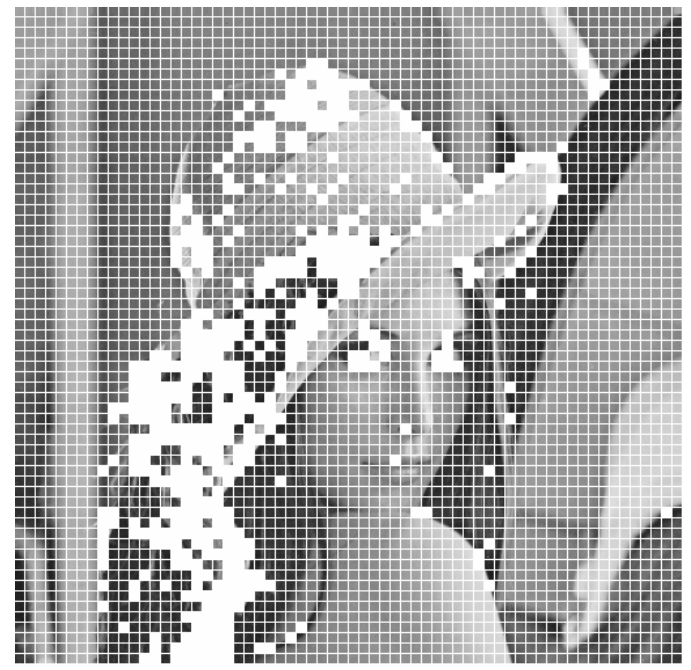

Figure 5. The Embedding Location of Lena

From figure 5, our proposed data hiding algorithm will mainly choose the blocks with low variance (smooth region). For the blocks with $45^{\circ}$ edge or highly texture properties, they will not be used for data hiding.

\section{EXTENSION TO LOSSLESS WATERMARKING}

In this section, we present how to extend the proposed lossless data hiding algorithm in delta domain to suit for the purpose of lossless watermarking. As our algorithm basically embeds information in smooth or low-variance blocks, our algorithm can be extended to withstand the JPEG attack (JPEG compression is always modeled as a particular low-pass operation). By storing the location map of watermarking to the authorized third party, the watermark can be extracted from a possibly corrupted image with the help of location map. The performance of test images against JPEG attack is shown in figure 6.

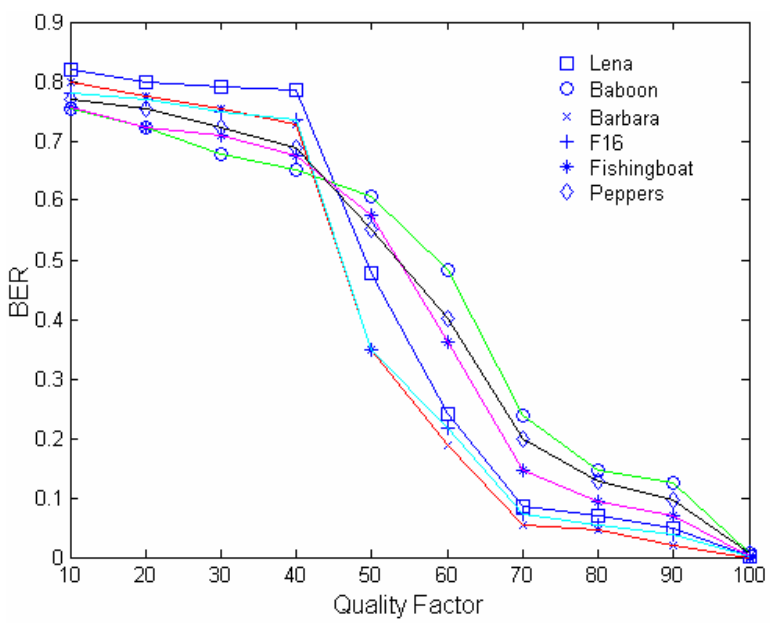

Figure 6. BER of Test Images Against JPEG Attack

From figure 6 , when the quality factor is greater than 60 , the BER is less than 0.5, which means the watermark is still detectable For some cases, such as F16 and Barbara, our proposed algorithm can withstand up to quality factor of 50 .

\section{CONCLUSION}

In this paper, we propose a new novel lossless data hiding algorithm in delta domain. The MSE discount is obtained by performing watermarking in the proposed delta domain using the checkerboard-pattern watermark sequences. The watermark is embedded into a generally smooth region and the edges are preserved. The PSNR is high and there is no "salt-and-peppers" artifact. The proposed algorithm can be extended for lossless watermarking purpose to withstand the JPEG attack.

\section{ACKNOWLEDGEMENT}

This work has been supported by the Innovation and Technology Commission of the Hong Kong Special Administrative Region, China (project no. ITS/122/03 and project no. GHP/033/05).

\section{REFERENCES}

[1] N. Nikolaidis and I.Pitas, "Copyright protection of images using robust digital signatures" Proc. Int. Conf. Acoustics, Speech and Signal Processing, vol. 4, pp. 2168-2171, May 1996

[2] I. Cox, Kilian, T. Leighton, and T. Shamoon, "Secure spread spectrum watermarking for images, audio, and video" Proc Int. Conf. Image Processing, vol. 3, pp. 243-246, Sep. 1996

[3] C.W. Honsinger, P. Jones, M. Rabbani, and J. C. Stoffel, "Lossless recovery of an original image containing embedded data", $U$. S patent applicat., Docket No 77102/ E-D, 1999

[4] C. De Vleeschouwer, J.-F. Delaigle, B. Macq, "Circular interpretation of bijective transformations in lossless watermarking for media asset management", IEEE Trans. On Multimeida, vol. 5, no. 1, Mar, 2003

[5] Tian, J., "High capacity reversible data embedding and content authentication", IEEE Int. Conf. On Acoustics, Speech, and Signal Processing, vol. 3, pp. 517-520, April 2003 